\title{
"VIABILIDAD DE REDUCCIÓN DE EMISIONES POR DEFORESTACIÓN Y DEGRADACIÓN DE BOSQUES: EL CASO DE LAS ÁREAS NATURALES PROTEGIDAS DEL PERÚ"
}

\author{
Augusto Castro N. ${ }^{1}$, Roger Loyola G. ${ }^{2}$
}

Fecha de recepción: 10-10-12

\section{Resumen}

El Perú cuenta con 75 Áreas Naturales Protegidas (ANP) las cuales operan en condiciones financieras que no permiten la implementación de sus planes maestros de gestión. En este contexto, REDD+ constituye una alternativa de acceso a recursos financieros para la gestión de las ANP. El presente estudio evaluó la viabilidad de la implementación del mecanismo REDD+ en ANP seleccionadas del Sistema Nacional de Áreas Naturales Protegidas por el Estado. El Análisis concluye que el número de ANP en las que sería viable la implementación de proyectos REDD+, según las condiciones actuales del mercado voluntario de carbono forestal, fluctúa apenas entre 2 y 7 .

Palabras Clave: REDD+, costo beneficio, Áreas Naturales Protegidas.

Clasificación JEL: Q23
Fecha de aceptación: 27-08-13

\section{Abstract}

Peru has 75 Natural Protected Areas (ANP) which operate under financial conditions that don't allow the implementation of its management plans. In that context, REDD+, constitutes an option to bring financial resources to ANP. The present research assessed REDD+ implementation in selected ANP from the Natural Protected Areas National System (SINAMPE). The analysis concluded, that under the current status of the forest voluntary carbon markets, REDD+ implementation would be viable just between 2 and 7 ANP.

Key words: REDD+, cost benefit, Natural Protected Areas

JEL Classification: Q23

\section{INTRODUCCIÓN}

Se estima que cada año se pierden 13 millones de ha de bosques tropicales a nivel global

2 Doctor en Planeamiento Energético (Universidad Federal de Rio de Janeiro - Brasil). Profesor en economía ambiental y valoración ecológica (UNALM - Perú). Director de Evaluación, Valoración y Financiamiento del Patrimonio Natural (MINAM). Dirección postal: Av. La Universidad s/n La Molina Perú. Teléfono: (511) 6147800 anexo: 239; e-mail: rogerloyola@lamolina.edu.pe 
emitiendo a la atmósfera entre 5.6 y $8.6 \mathrm{Gt}$ de carbono (Kremen, Niles et al. 2000, Bellassen and Gitz 2008), cifras que se encontrarían ene tre el $12 \%$ y el $17 \%$ de las emisiones totales de $\mathrm{CO}_{2}$ (Kremen, Niles et al. 2000, Bellassen and Gitz 2008, Van der Werf, Morton et al. 2009, Burgess, Bahane et al. 2010). Ante este hecho, la Convención Marco de las Naciones Unidas para el Cambio Climático (UNFCCC por sus siglas en inglés) discute sobre los medios y elementos necesarios para frenar este proceso (UNFCCC 2009, UNFCCC 2010, UNFCCC 2011). Entre las alternativas discutidas para asegurar el éxito en la lucha contra el cambio climático, se encuentra la implementación de un mecanismo para la reducción de emisiones provenientes de la deforestación y de la degradación de los bosques en países en desarrollo (mecanismo comúnmente conocido como REDD+), que implica, además de la reducción de la deforestación y la degradación de los bosques, el manejo forestal sostenible y el incremento y conservación de las reservas de carbono de los bosques.

REDD+ es considerado, en el informe sobre la economía del cambio climático (Stern 2007), como el mecanismo más costo efectivo para abordar la mitigación del cambio climático. Además, es una alternativa significativa, efectiva, rápida y "win-win" (gana-gana) para desacelerar las tasas de deforestación y degradación forestal, para promover la conservación de los bosques, para contribuir al fortalecimiento de la gobernanza forestal y para apoyar en los esfuerzos de lucha contra la pobreza y desarrollo económico sostenible (Angeld sen 2008). Adicionalmente, si se reducen las emisiones provenientes de la deforestación y de la degradación de los bosques es posible que se obtengan beneficios adicionales e.g. la conservación de la biodiversidad y de los conocimientos ancestrales de las comunidades que habitan los bosques (Turner, Brandon et al. 2007, Malhi, Roberts et al. 2008, Baker, Jones et al. 2010, Turner, Brandon et al. 2012).

Perú es considerado como un país con grandes extensiones de bosques y baja tasa de deforestación (da Fonseca, Rodriguez et al. 2007). Asimismo, es el segundo en superfip cie de bosques tropicales en América Latina, y el cuarto a nivel mundial. Posee más de 70 millones de hectáreas de bosques tropicales y el $13 \%$ de los bosques amazónicos. Se estima que entre los años 1990 y 2000 se deforestaron anualmente 150,000 ha por año (MINAM 2010). Sin embargo, las amenazas de deforestación y degradación forestal se viene incrementando en los últimos años, en parte como consecuencia de un crecimiento económico acelerado, basado principalmente en la explotación de recursos naturales (MINAM 2011).

Se sabe, además, que en la actualidad los cambios en el uso del suelo y las prácticas en el sector forestal son la principal causa de las emisiones de GEl en el Perú, pues aporta el 47\% de las emisiones nacionales (MINAM 2010). En ese sentido, el estado, apoyado por la sociedad civil, está promoviendo e implementando acciones a nivel nacional, regional y local para recuperar y conservar los ecosistemas forestales e.g. Programa Nacional de Conservación de Bosques para la Mitigación del Cambio Climático. Sin embargo, estas acciones requieren de mayor soporte técnico y financiero para 
alcanzar la meta voluntaria, presentada por el Perú, de reducir su deforestación neta en bosques naturales a cero al año 2021. En especial, si se considera que es altamente probable que la actual tasa de deforestación se incremente en las próximas décadas, dados los planes de inversión existentes y la presión de actividades ilícitas que implican deforestación y degradación de bosques.

En las Áreas Naturales Protegidas (ANP) del Perú, el cambio en el uso de suelo no está permitido por lo que la amenaza de deforestación y degradación de bosques no debiera existir. Sin embargo, estos espacios no cuentan con los recursos financieros necesarios para su gestión (Villanueva 2005) con lo que se justifica la necesidad de buscar alternativas adicionales que contribuyan a su preservación.

En ese contexto, REDD+ constituye una alternativa de recursos financieros para la gestión de las ANP; al mismo tiempo, para contribuir con los objetivos globales de reducción de emisiones de $\mathrm{GEl}$, conservar la biodiversidad y disminuir la pobreza en zonas de amortiguamiento de ANP. Dada la situación de déficit de recursos financieros, bajo la que se gestionan las ANP, en el presente estudio se evalúa si el mecanismo REDD+ constituye una alternativa viable para proveer recursos financieros para la gestión de las ANP. Con esta finalidad, se analizarán cuatros aspectos fundamentales: 1) ingresos por venta de certificados de reducciones voluntarias de emisiones (VERs) producidos por actividades de REDD+ en ANP seleccionadas; 2) costos de gestión según tamaño de ANP y para los escenarios de recursos financieros óptimo y mínimo; 3) re- lación costo beneficio para proyectos REDD+ en ANP seleccionadas; 4) priorización de ANP como parte de una estrategia REDD+ de alcance nacional.

Las expectativas sobre altas rentabilidades de REDD+ han sido construidas basadas en análisis de costos de oportunidad (Wertz-Kanounnikoff 2008) que generalmente no cona sideran otras categorías de costos, como son los costos de transacción y los costos de implementación (Pagiola and Bosquet 2009). En estos estudios, se considera a los costos de oportunidad como los más elevados (Irawan, Tacconi et al. 2013), Sin embargo, recientes estudios sugieren que las otras categorías de costos no son irrelevantes (Tacconi 2012). En el presente trabajo, por un lado, se consideran los costos de implementación de REDD+ como la principal categoría de costos; por otro, se reconoce que cuando se habla de REDD+ en la UNFCCC se hace referencia a acciones principalmente enmarcadas en el nivel nacional para reducir las emisiones de gases de efecto invernadero provenientes de la deforestación y degradación de bosques y conservar e incrementar los stocks de carbono. Sin embargo, las estimaciones y resultados del presente trabajo de investigación consideran como acciones REDD+ aquellas que buscan el mercado voluntario de carbono; $y$ están dirigidas, principalmente, a evitar deforestación.

En la sección II, se presentan los antecedentes de REDD+, los costos relacionados con su implementación y trabajos sobre la economía de REDD+ realizados previamente. En la sección III y IV, se plantea la metodología; y 
se discuten los resultados, respectivamente. Finalmente, en la sección $\mathrm{V}$, se concluye sobre la viabilidad de implementación de REDD+en ANP, y se discute la pertinencia de usar el mecanismo como una herramienta complementaria a los esfuerzos de conservación actuales.

\section{ANTECEDENTES}

\subsection{Contexto internacional REDD+}

La discusión sobre la reducción de emisiones en el sector forestal se inició en el año 2005 durante la décimo primera sesión de la Conferencia de las Partes (COP 11) de la UNFCCC. En esa oportunidad; Papúa, Nueva Guinea y Costa Rica presentaron una propuesta para considerar opciones con el fin de reducir emisiones de GEl ocasionadas por la deforestación - RED (Hiraldo and Tanner 2011). El argumento utilizado fue que ni la UNFCCC ni el protocolo de Kioto consideraban las emisiones provenientes de la deforestación y que para esa fecha se estimaban que eran equivalentes a por lo menos el $17 \%$ de las emisiones globales totales. Esa propuesta constituye el primer intento de incluir la "deforestación evitada" como parte de un acuerdo climático de carácter global.

A la fecha, se ha aumentado de manera considerable el alcance de la propuesta inicial de Papúa, Nueva Guinea y Costa Rica. En la COP 11 , se consideró que la degradación de los bosques, principalmente por tala selectiva, constituye una causa importante de emisiones de GEI en países en desarrollo, y se solicitó al Órgano Subsidiario de Asesoramiento Técnico y Científico (SUBSTA por sus siglas en inglés) que realice consultas y acciones necesarias para evaluar la viabilidad de REDD, con lo cual se incluyen las emisiones provenientes de la degradación de bosques en el mecanismo.

El Plan de Acción de Bali, decidido en la COP 13 del año 2007, señala que los enfoques para mitigar el cambio climático deben incluir: "temas referentes a la reducción de emisiones por deforestación y degradación de bosques en países en desarrollo; así como el rol de la conservación, manejo sostenible de los bosques y el mantenimiento de los stocks de carbono en los países en desarrollo" (Hiraldo and Tanner 2011). Sin embargo, no es hasta la COP 15 cuando se empieza a hablar de REDD+ y se refiere a: "medidas de políticas e incentivos positivos en temas referentes a la reducción de emisiones por deforestación y degradación de bosques; así como el rol de la conservación, manejo sostenible de los bosques y el mantenimiento de los stocks de carbono en los países en desarrollo"(UNFCCC 2009).

En cuanto a medios de implementación del mecanismo REDD+, en la COP 15, realizada en diciembre del año 2009, se esperaba alcanzar resultados sobre los detalles que permitirían la amplia aplicación de REDD+. Las discusiones se centraron, principalmente, en el acceso a recursos financieros y la escala de implementación; sin embargo no fue posible alcanzar consensos entre las partes (UNFCCC 2009). Fue recién en la COP 16 realizada en din ciembre del 2010 donde se tomaron decisiones sobre las acciones que deben realizar los 
países en desarrollo que, voluntariamente, deseen implementar el mecanismo: (1) escenarios de referencia; (2) sistemas de medición, reporte y verificación de gases de efecto invernadero; (3) estrategias nacionales REDD+; y (4) sistemas para informar cómo las salvaguardas sociales y ambientales están siendo consideradas en las acciones REDD+ (UNn FCCC 2010). Asimismo, se llegó a un consenso acerca de las 3 fases para la implementación de REDD+ (Angelsen, Brown et al. 2009); y se decidió que los escenarios de referencia y los sistemas de medición, reporte y verificación podrían desarrollarse de manera interina a escala sub nacional mientras que los países incrementan sus capacidades para alcanzar el nivel nacional (UNFCCC 2010).

REDD+ es un mecanismo financiero aún bajo discusión en la UNFCCC; y, por lo tanto, a la fecha no existe un mercado regulado por las Naciones Unidas de Certificados de Reducciones de Emisiones de Carbono proveniente de REDD+. Sin embargo, existen mercados voluntarios (no regulados por la UNFCCC) que buscan compensar acciones REDD+ (Streck 2012). Estos mercados seguirán en funcionai miento mientras no se tomen decisiones en la UNFCCC. Las reglas de estos mercados voluntarios de carbono forestal serán detalladas durante el desarrollo del trabajo de investigación, y se usarán como referencia para la realización de los cálculos del análisis.

\subsection{Costos y beneficios de REDD+}

REDD+ fue propuesto para incentivar a los países en desarrollo la implementación de acciones para reducir emisiones de la deforestación y conserven sus bosques con la expectativa que los pagos de carbono superarían los costos necesarios para alcanzar el objetivo de reducir la deforestación y la degradación de bosques (12). Los costos de REDD+ incluyen los costos de implementación, los costos de oportunidad y los costos de transacción. Los costos de implementación emergen de las actividades necesarias para hacer frente a las causas de deforestación. Los costos de oportunidad son los beneficios de la mejor alternativa productiva que se dejarían de percibir como resultado de REDD+. Diversos trabajos sobre los costos y beneficios de REDD+ han sido publicados recientemente [ver por ejemplo (Kremen, Niles et al. 2000, Borner and Wunder 2008, Nepstad, Soares-Filho et al. 2009, Börner, Wunder et al. 2010, Hunt 2010, Merger, Held et al. 2012, Irawan, Tacconi et al. 2013)]. Estos estudios consideran a los costos de oportunidad como los más altos (28); $y$, por consiguiente, los más relevantes para el análisis; constituye, por ende, en atención de un gran número de estudios. Los costos de transacción están referidos a los procesos de negociación necesarios para la firma de acuerdos y arreglos institucionales. Las expectativas sobre la rentabilidad del mecanismo fueron construidas principalmente, basadas, en estudios de costos de oportunidad. Estos estudios, por lo general, no consideran los otros costos de REDD+ como son los costos de transacción y los costos de implementación (27). Sin embargo, los otros costos no son irrelevantes $(10,30)$ y por ello es posible que estos estudios sobreestimen la rentabilidad de REDD+. 


\section{MÉTODOS}

\section{1. Área de estudio}

\subsubsection{Las áreas protegidas del Perú}

Las áreas protegidas del Perú pueden clasificarse, de acuerdo a quien las administra, en tres grupos: Las Áreas Naturales Protegidas que pertenecen al Sistema Nacional de Áreas Naturales Protegidas por el Estado (SINANPE) y son administradas por el gobierno nacional; las áreas de conservación regionales (ACR), administradas por los gobiernos regionales; y las áreas de conservación privadas (ACP), administradas por personas particulares $\mathrm{O}$ empresas privadas en coordinación con el gobierno.

\subsubsection{Sistema Nacional de Áreas Naturales Protegidas por el Estado}

EI SINAMPE se encuentra bajo la jurisdicción del Servicio Nacional de Áreas Naturales Protegidas por el Estado (SERNANP), entidad bajo jurisdicción del Ministerio del Ambiente. Antes de la creación del Ministerio del Ambiente, se hallaba bajo jurisdicción del Ministerio de Agricultura a través del Instituto Nacional de Recursos Naturales (INRENA). En agosto del 2012, el SINANPE estaba conformado por 75 ANP (19,5 millones de ha). Adicionalmente a estas Áreas protegidas, en agosto del 2012 se habían conformado 15 ACR (2,4 millones de ha) y 51 ACP (196 480,86 ha) con lo que el territorio total protegido del país es igual a 22,1 millones de ha $(16,91 \%$ de la superficie del Perú).
EI SINANPE cuenta con nueve categorías de ANP definitivas que, según su condición legal, finalidad y usos permitidos pueden ser ANP de uso directo y ANP de uso indirecto. Además de las 9 categorías mencionadas, se encuentran dos categorías en estudio: El Área de conservación Privada y la Zona Reservada.

Las Zonas Reservadas, se establecen de forma transitoria en áreas que, reuniendo las condiciones para ser consideradas como áreas naturales protegidas, requieren de estudios complementarios.

\subsubsection{Casos de estudio}

La selección de los casos de estudio se realizó en dos etapas: 1) preselección de 40 ANP (16,5 millones de ha), de las 75 ANP del SINANPE. Ésta se basó en la disponibilidad de información cartográfica; 2) selección final de las 25 ANP (14,3 millones de ha) que presentaron variaciones en la superficie de bosques (deforestación) al año 2050, según información disponible en Soares-Filho et.al. (2006). En la Tabla 1, se presentan las 25 ANP seleccionadas, categoría, ubicación, superficie y clasificación según tamaño.

\subsection{Ingresos por ventas en el mercado voluntario de carbono}

Para estimar potenciales ingresos por ventas de VERs, se utilizó la siguiente formula:

$$
I=E G E I^{*} \operatorname{Pr}^{*} E f
$$




\section{Tabla 1. 25 ANP seleccionadas}

\begin{tabular}{|c|c|c|c|c|}
\hline ANP & Categoría & $\begin{array}{c}\text { Ubicación } \\
\text { (Departamento) }\end{array}$ & $\begin{array}{l}\text { Superficie } \\
\text { total (ha) }\end{array}$ & Tamaño \\
\hline Cordillera Azul & Parque Nacional & $\begin{array}{l}\text { Huancavelica, Loreto, San Martin, } \\
\text { Ucayali }\end{array}$ & $1,353,190.85$ & Grande \\
\hline Sierra del Divisor & Zona Reservada & Loreto, Ucayali & $1,478,311.39$ & Grande \\
\hline Alto Mayo & Bosque de Protección & San Martín & $177,749.84$ & Grande \\
\hline Amarakaeri & Reserva Comunal & Cuzco, Madre de Dios & $402,335.62$ & Grande \\
\hline Tambopata & Reserva Nacional & Madre de Dios & $274,690.88$ & Grande \\
\hline $\begin{array}{l}\text { Ichigkat Muja-Cordillera } \\
\text { del Cóndor }\end{array}$ & Parque Nacional & Amazonas & $88,477.00$ & Mediana \\
\hline Cordillera de Colan & Santuario Nacional & Amazonas & $39,215.80$ & Mediana \\
\hline Santiago Comaina & Zona Reservada & Amazonas Loreto & $398,449.44$ & Grande \\
\hline El Sira & Reserva Comunal & Cuzco, Huancavelica, Pasco & $616,413.41$ & Grande \\
\hline BahuajaSonene & Parque Nacional & Madre de Dios & $1,091,416.00$ & Grande \\
\hline San Matías-San Carlos & Bosque de Protección & Pasco & $145,818.00$ & Grande \\
\hline Manu & Parque Nacional & Cuzco, Madre de Dios & $1,716,285.22$ & Grande \\
\hline ChayuNaín & Reserva Comunal & Amazonas & $23,597.76$ & Mediana \\
\hline Pampa Hermosa & Santuario Nacional & Junín & $11,543.74$ & Mediana \\
\hline Yanachaga-Chemillén & Parque Nacional & Pasco & $110,657.78$ & Grande \\
\hline Gueppy & Zona Reservada & Loreto & $613,682.61$ & Grande \\
\hline Pagaibamba & Bosque de Protección & Cajamarca & $2,031.02$ & Pequeña \\
\hline Río Abiseo & Parque Nacional & San Martin & $272,407.96$ & Grande \\
\hline PuiPui & Bosque de Protección & Junín & $53,467.21$ & Mediana \\
\hline Megantoni & Santuario Nacional & Cuzco & $215,868.96$ & Grande \\
\hline Pacaya Samiria & Reserva Nacional & Loreto & $2,170,247.45$ & Grande \\
\hline Tingo María & Parque Nacional & Huánuco & $4,777.50$ & Pequeña \\
\hline Alto Purús & Parque Nacional & Madre de Dios, Ucayali & $2,514,775.23$ & Grande \\
\hline Huascarán & Parque Nacional & Ancash & $340,002.62$ & Grande \\
\hline Machiguenga & Reserva Comunal & Cuzco & $218,905.63$ & Grande \\
\hline
\end{tabular}

Fuente: SINAMPE.

Donde:

\section{I: Ingreso por venta de VERs en US\$}

EGEI: Emisiones de gases de efecto invernadero en $\mathrm{tCO}_{2} \mathrm{e}$

Pr: Precio negociado para los VERs

Ef: Eficiencia de las actividades REDD+ implementadas.
La variable EGEI fue estimada haciendo uso de herramientas SIG y basados en proyecciones de deforestación y en mapas de distribución de biomasa viva sobre el suelo en la Amazonia. Con ello, se estimaron las emisiones de GEl proyectadas al año 2050 bajo el escenario BAU para las ANP seleccionadas.

El trabajo se realizó en tres etapas: En la eta- 
pa (1), se determinó la superficie deforestada cada 5 años (2010 - 2050) bajo un escenario BAU haciendo uso de los resultados de Soares-Filho et al. (2006). En las Figuras 1 y 2 , se muestran la evolución de la deforestación entre los años 2010 y 2050 para el escenario $\mathrm{BAU})$. En la etapa (2), se relacionó la superficie deforestada con el contenido de $\mathrm{CO}_{2}$ haciendo uso de los resultados presentados por Saatchi et al. (2007). En la Figura 3, se puede ver la distribución de contenido de $\mathrm{CO}_{2}$ para la amazonia peruana. Durante la etapa (3), se determinaron las emisiones en $\mathrm{tCO}_{2}$ e bajo el escenario BAU calculando el producto de la superficie deforestada con el contenido de $\mathrm{tCO}_{2}$ e relacionado a esa deforestación. En el Anexo 1, se muestra, como ejemplo, el proceso seguido para estimar las emisiones de GEI para el Bosque de Protección Alto Mayo.

\section{Figura 1. Deforestación al 2010, escenario BAU}

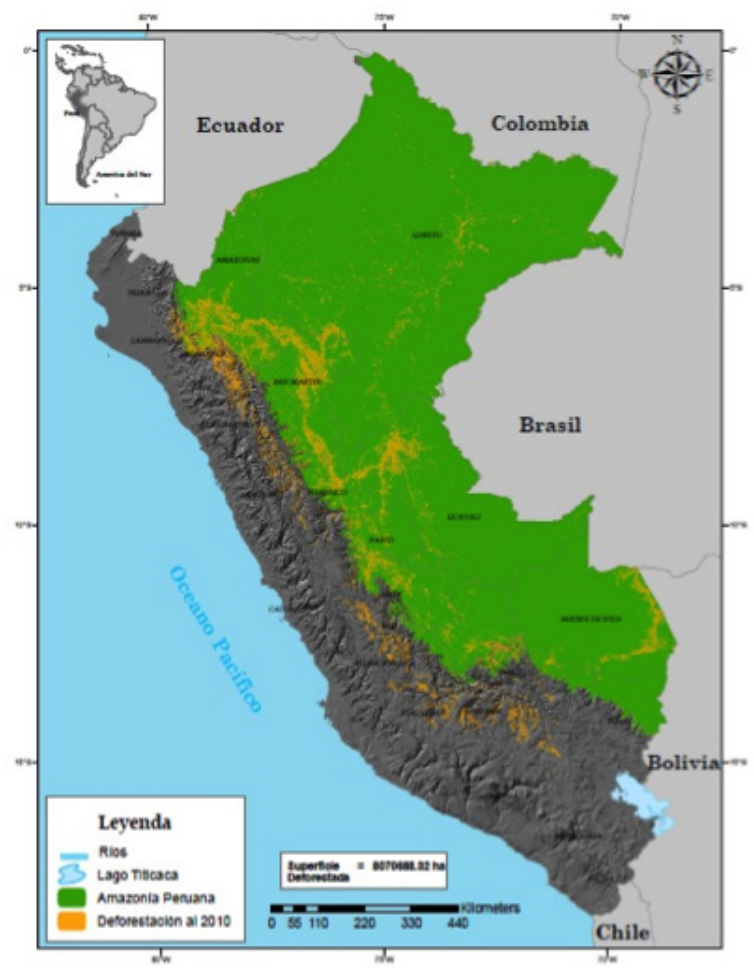

Fuente: Soares-Filho et al. (2006). 
Figura 2. Deforestación al 2050 escenario BAU

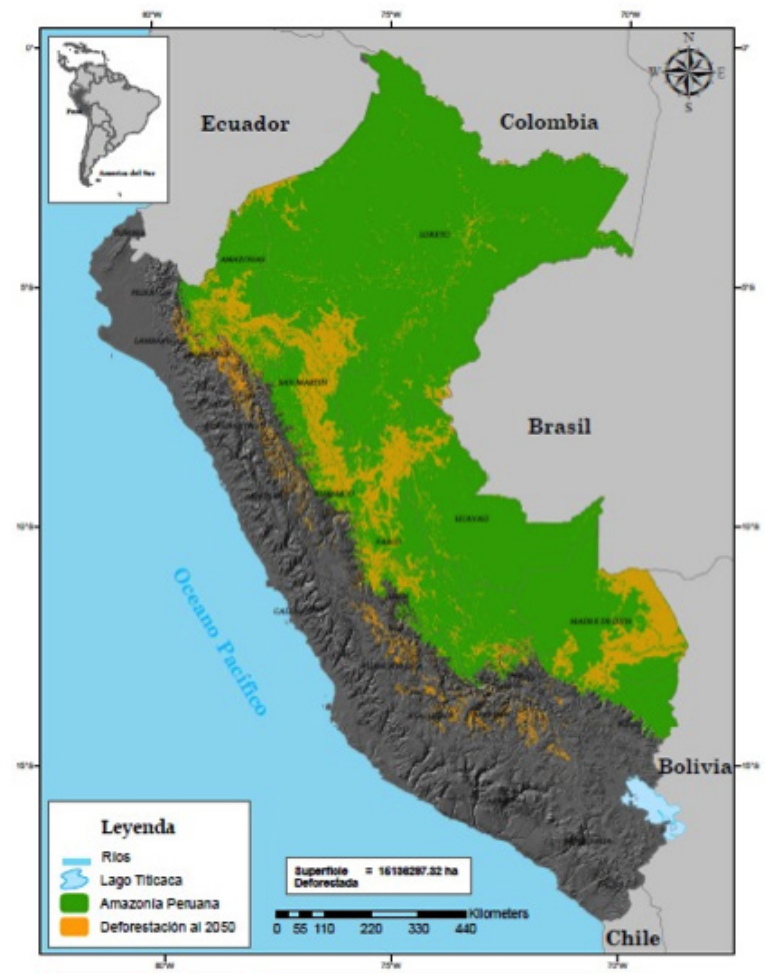

Fuente: Soares-Filho et al. (2006).

Figura 3. Mapa distribución biomasa aérea en la Amazonia peruana ( $\mathrm{tCO}_{2} \mathrm{e} / \mathrm{ha}$ )

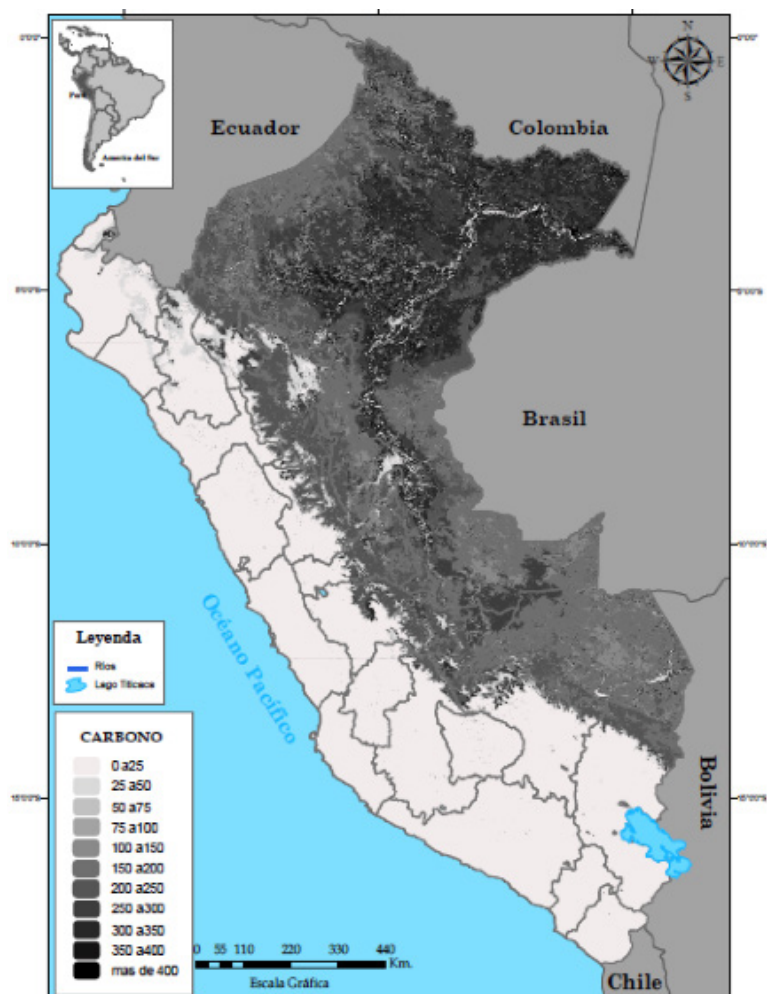

Fuente: Saatchi et al. (2007). 
La variable $\mathrm{Pr}$, es equivalente al precio promedio negociado en el mercado voluntario de carbono forestal entre los años 2008, 2009 y 2010 equivalente US\$ 5.4 (Diaz, Hamilton et al. 2011). Adicionalmente, se realizaron estif maciones de ingresos con un precio arbitrario de US\$ 10.0 .

Para determinar Ef, se definieron 2 escenarios bajo el supuesto que las acciones REDD+ pueden ser $100 \%$ o $75 \%$ efectivas dependiendo de las inversiones realizadas según la disponibilidad de acceso a recursos financieros. Un escenario de acceso a recursos financieros óptimos permitiría, entonces, implementar acciones REDD+ $100 \%$ efectivas Ef (100\%). Mientras que el acceso a recursos financieros mínimos permitiría implementar acciones REDD+ 75\% efectivas Ef (75\%).

Adicionalmente, se tomaron las siguientes consideraciones para estimar los ingresos por ventas de VERs: (i) el período de compromiso después del cual la deforestación es medida y las compensaciones son recibidas es igual a 5 años; (ii) los beneficios adicionales obtenidos de la manutención de ANP (e.g. servicios ambientales $\mathrm{u}$ otros bienes y servicios del bosque) no son considerados en el análisis; (iii) los supuestos y características descritas para los trabajos realizados por Soares-Filho et al. (2006) y Saatchi et al (2007).

\subsection{Costos REDD+ en ANP}

Los costos de implementar REDD+ en ANP fueron estimados mediante la siguiente formula:

$$
C=C I+C O+C T
$$

Donde:

C: costo total de implementar REDD+ en un ANP

Cl: costo de implementación de las actividades REDD+

CO: costo de oportunidad

CT: costo de transacción.

Los costos de implementación $(\mathrm{Cl})$ fueron estimados mediante la siguiente fórmula:

$$
C I=C G * \text { Superficie del ANP }
$$

Donde:

CG es equivalente a los costos actualizados de gestión de ANP en US\$/ha. Estos costos fueron reportados por Villanueva (2005) para los escenarios: (i) de acceso a recursos financieros óptimos, el cual supone que un ANP cuenta con los medios para un manejo conforme a su plan maestro; (ii) de acceso a recursos financieros mínimos, el cual supone que un ANP tiene ciertos recursos y medios indispensables para su manejo, pero que le faltan elementos para alcanzar un nivel de recursos financieros óptimo.

Para actualizar los costos de gestión al 2010, según tamaño de ANP y para los escenarios propuestos, se: (1) convirtieron los valores en US\$ propuestos por Villanueva (2005) a nuevos soles utilizando un tipo de cambio de $3.423 \mathrm{~S} /$. / US\$; (2) se afectaron los valores obtenidos por la tasa de inflación anual según 
datos del Instituto Nacional de Estadísticas e Información (INEI); (3) se convirtieron los valores obtenidos en S/. a US\$ usando el tipo de cambio de 2.815 S/./US\$ a diciembre del 2010.

Asimismo, para estimar el costo total de implementar REDD+ en un ANP se tuvieron en cuenta los siguientes supuestos: (i) los costos de elaboración de escenarios de referencia de emisiones y del establecimiento de un sistema para medir, reportar y verificar las reducciones de emisiones son asumidos por el nivel nacional; (ii) los costos por ha de mantener las ANP se mantienen constantes en el tiempo; (iii) el estado peruano asumió los costos de oportunidad de impedir el cambio de uso del suelo en las ANP declaradas a la fecha.

Bajo esos supuestos el costo total de implementar REDD+ en un ANP (C) sería equivalente al costo de implementación de las actividades REDD+ (CG). Sin embargo, se consideró conveniente incluir un escenario que comprenda tanto los costos de oportunidad, como los costos de transacción. Para la construcción de ese escenario, se utilizaron los siguientes valores: El costo de oportunidad (CO) fue tomado del costo de oportunidad promedio por ha, valor reportado por Armas et.al. (2009) equivalente a 4361 S/./ha (1515.82 US\$/ha) para la amazonia peruana. Los costos de transacción fueron tomados del rango de valores reportados por Chenost y Gardette (2009) que oscilan entre US\$ 172,000 y US\$ 878000 por proyecto, este valor incluye los costos de elaboración de los documentos de proyecto. Para el presente estudio se tomó el valor inferior, equivalente a US\$172,000.

\subsection{Relación Costo Beneficio}

La relación costo beneficio $(B / C)$ fue estimada calculando la proporción entre el valor presente neto (VPN) de: (i) los flujos estimados de ingresos por ventas de VERs bajo los escenarios de $100 \%$ y $75 \%$ de efectividad de las actividades REDD+; y (ii) el costo de gestión según tamaño de las ANP seleccionadas bajo los escenarios de acceso a recursos financieros óptimos y mínimos. El análisis se realizó utilizando dos tasas sociales de descuento. Una equivalente al 10\% es usada para proyectos sociales por el Ministerio de Economía y Finanzas del Perú. La otra, equivalente al $5 \%$, considerada como apropiada por estudios similares en otras regiones del mundo (Bellassen and Gitz 2008).

\section{RESULTADOS Y DISCUSIÓN}

\subsection{Ingresos por venta de VERs}

Los resultados del análisis confirman que el tamaño del ANP no es una variable que determine el nivel de ingresos y, por el contrario, las reservas de carbono y las amenazas de deforestación son variables determinantes en el nivel de ingresos de una iniciativa REDD+ (Miles and Kapos 2008), sobre todo cuando el énfasis de los proyectos en el mercado voluntario de carbono se centra en la Reducción de la Deforestación (RED) lo que disminuye la atención, por cuestiones técnicas, en los otros componentes de REDD+ como son: la degradación de bosques, los incrementos de los stocks de carbono, el manejo forestal sostenible y la conservación de los stocks de carbono (Ebea ling and Yasué 2008). 
Las 5 ANP que presentan mayores EGEl totales para los años 2010-2050 son: Cordillera Azul, Sierra del Divisor, Alto Mayo, Amarakaeri y Tambopata (ver Anexo 2). Asimismo, el valor promedio de ingresos por venta de VERS para el escenario $\mathrm{Ef}(100 \%)$ y $\mathrm{Pr}=5.4$ US\$, sería equivalente a US\$ $63,471,390$, con un valor máximo de ingresos totales de US\$245,310,111 para el Parque Nacional (PN) Cordillera Azul y un mínimo de US\$ 0 para la Reserva Comunal (RC) Machiguenga. Dada la relación entre los ingresos por venta de VERS y EGEI, ingresos equivalentes a US\$ 0 (e.g los obtenidos por la RC Machiguenga) son explicados por la escasa deforestación que esta ANP presentaría.

Para el escenario Ef (75\%) y $\mathrm{Pr}=$ US\$ 5.54; el valor promedio de los ingresos totales es equivalente a US\$ 47,603,541; con un valor máximo de US\$183,982,583 y un mínimo equivalente a US\$ 0 . Cambios significativos en los potenciales ingresos por ventas de VERs fueron hallados al variar el nivel de efectividad de las actividades REDD+ que se traducen en mayores EGEI evitadas.

Las ANP con valores máximos y mínimos de ingresos, bajo cualquiera de los escenarios, fueron PN Cordillera Azul y RC Machiguenga respectivamente.

\subsection{Costos REDD+ en ANP del Perú}

Si bien los costos para evitar la deforestación varían según ANP, estas variaciones no están relacionadas con variaciones potenciales en EGEI (Miles and Kapos 2008). Del análisis, se puede inferir que estas variaciones están relacionadas con el tamaño de las áreas y la capacidad de las instituciones proponentes de aprovechar economías de escala. Esto hace suponer que quizás sea más rentable implementar REDD+ en ANP de tamaño grande o mediana y que será necesario buscar mecanismos financieros adicionales para lograr el mantenimiento integral y total de las ANP al interior del SINANPE.

Para el escenario recursos financieros óptimos, el $\mathrm{Cl}$ promedio es equivalente a US\$ 96,042,215, el máximo US\$352,561,928 para PN Alto Purus y el mínimo USS 15,513,800 para el PN Yanachaga-Chemillen. Bajo el escenario de acceso a recursos financieros mínimos, el $\mathrm{Cl}$ promedio de un ANP es equivalente a US\$ $59,336,620$. Con valores máximos y mínimos de US\$224,488,411 y US\$ 7,925,105 respectivamente y correspondientes, nuevamente, a los PN Alto Purus y Yanachaga-Chemillen.

\section{Tabla 2. Costos de gestión en US\$/ha según tamaño de ANP actualizados al año 2010}

\begin{tabular}{|c|c|c|}
\hline Tamaño ANP & Recursos óptimos & Recursos mínimo \\
\hline Grande & 3.42 & 2.18 \\
\hline Mediana & 40.38 & 22.23 \\
\hline Pequeña & 231.56 & 95.17 \\
\hline
\end{tabular}

Fuente: Elaboración propia con base en Villanueva (2005). 


\subsection{Relación costo beneficio de REDD+ en ANP del Perú}

Los valores de la relación $B / C$ para el escenario de Ef (100\%) y Pr US\$ 5.4 muestran que sería viable implementar REDD+ sólo en las 2 ANP que poseen valores de relación $B / C$ mayores a 1: Bosque de Protección (BP) Alto Mayo y la Reserva Nacional (RN) Tambopata.
Para el resto de las ANP, el valor de la relación $B / C$ es menor a uno con lo que se haría inviable la implementación de REDD+. Valores similares fueron obtenidos cuando: (i) la tasa de descuento utilizada fue del $5 \%, \operatorname{Pr}=$ US\$ 5.4 y Ef (100\%); (ii) y cuando tasa de descuento utilizada fue $10 \%, \operatorname{Pr}=$ US\$5.4 y Ef (75\%); bajo esos escenarios sería viable implementar actividades REDD+, en las mismas dos ANP mencionadas anteriormente (ver Tabla 3).

Tabla 3. Valores de la relación B/C para Pr = US\$ 5.4 (100 y 75 \% efectividad)

\begin{tabular}{|c|c|c|c|c|}
\hline \multirow[b]{2}{*}{ ANP } & \multicolumn{2}{|c|}{$\mathrm{B} / \mathrm{C}(100 \%)$} & \multicolumn{2}{|c|}{$\mathrm{B} / \mathrm{C}(75 \%)$} \\
\hline & $\begin{array}{c}\text { Tasa Descuento } \\
10 \%\end{array}$ & $\begin{array}{c}\text { Tasa Descuento } \\
5 \%\end{array}$ & $\begin{array}{c}\text { Tasa Descuento } \\
10 \%\end{array}$ & $\begin{array}{c}\text { Tasa Descuento } \\
5 \%\end{array}$ \\
\hline Cordillera Azul & 0.68 & 0.94 & 0.80 & 1.10 \\
\hline Sierra del Divisor & 0.41 & 0.65 & 0.49 & 0.78 \\
\hline Alto Mayo & 1.95 & 2.11 & 2.30 & 2.48 \\
\hline Amarakaeri & 0.77 & 0.95 & 0.91 & 1.14 \\
\hline Tambopata & 1.00 & 1.06 & 1.17 & 1.24 \\
\hline $\begin{array}{l}\text { Ichigkat Muja- Cordillera } \\
\text { del condor }\end{array}$ & 0.22 & 0.22 & 0.30 & 0.30 \\
\hline Cordillera de Colan & 0.27 & 0.37 & 0.36 & 0.51 \\
\hline Santiago Comaina & 0.50 & 0.46 & 0.59 & 0.42 \\
\hline El Sira & 0.18 & 0.20 & 0.22 & 0.24 \\
\hline Bahuaja Sonene & 0.11 & 0.12 & 0.13 & 0.15 \\
\hline San Matías San Carlos & 0.36 & 0.59 & 0.42 & 0.70 \\
\hline Manu & 0.01 & 0.03 & 0.01 & 0.04 \\
\hline Chayu Naín & 0.38 & 0.39 & 0.51 & 0.54 \\
\hline Pampa Hermosa & 0.06 & 0.09 & 0.08 & 0.12 \\
\hline Yanachaga-Chemillén & 0.06 & 0.10 & 0.07 & 0.11 \\
\hline Gueppy & 0.00 & 0.01 & 0.00 & 0.01 \\
\hline Pagaibamba & 0.06 & 0.05 & 0.11 & 0.10 \\
\hline Rio Abiseo & 0.00 & 0.01 & 0.00 & 0.01 \\
\hline Pui Pui & 0.00 & 0.00 & 0.00 & 0.00 \\
\hline Megantoni & 0.00 & 0.01 & 0.00 & 0.01 \\
\hline Pacaya Samiria & 0.00 & 0.00 & 0.00 & 0.00 \\
\hline Tingo María & 0.00 & 0.00 & 0.00 & 0.01 \\
\hline Alto Purus & 0.00 & 0.00 & 0.00 & 0.00 \\
\hline Huascarán & 0.00 & 0.00 & 0.00 & 0.00 \\
\hline Machiguenga & 0.00 & 0.00 & 0.00 & 0.00 \\
\hline
\end{tabular}

Fuente: Elaboración propia en base a los datos estimados. 
Adicionalmente, los resultados muestran que RC Amarakaeri y el PN Cordillera Azul poseen relaciones $\mathrm{B} / \mathrm{C}$ cercanos a uno. Por ello, la implementación de REDD+ podría ser viable al disminuir los $\mathrm{Cl}$. Asimismo, cuando la tasa de descuento utilizada fue del $5 \%$ los valores de la relación $\mathrm{B} / \mathrm{C}$ oscilan entre 2.48 y 0 . Bajo ese escenario los resultados muestran que sería viable implementar actividades REDD+ en 4 ANP: BP Alto Mayo, RN Tambopata, RC Amarakaeri y PN Cordillera Azul.
Además, se realizaron estimaciones asumiendo $\operatorname{Pr}=$ US\$ 10.00. En ese caso, para Ef (100\%), y tasa de descuento del $10 \%$, se obtienen valores de relación $B / C$ mayores a 1 en 4 de las 25 ANP analizadas: BP Alto Mayo, RN Tambopata, RC Amarakaeri, PN Cordillera Azul. Asimismo, si la tasa de descuento utilizada fuera de $5 \%$, los resultados muestran que la implementación de REDD+ sería viable, adicionalmente a las ANP mencionadas anteriormente, en las Zonas Reservadas (ZR) Santiago Comaina y Sierra Divisor y BP San Matías-San Carlos (ver Tabla 4).

Tabla 4. Valores de relación B/C para Pr = US\$ 10.00 (100 y 75 \% efectividad)

\begin{tabular}{|c|c|c|c|c|}
\hline \multirow[b]{2}{*}{ ANP } & \multicolumn{2}{|c|}{$B / C(100 \%)$} & \multicolumn{2}{|c|}{$B / C(75 \%)$} \\
\hline & $\begin{array}{c}\text { Tasa Descuento } \\
10 \%\end{array}$ & $\begin{array}{c}\text { Tasa Descuento } \\
\mathbf{5 \%}\end{array}$ & $\begin{array}{c}\text { Tasa Descuento } \\
10 \%\end{array}$ & $\begin{array}{c}\text { Tasa Descuento } \\
5 \%\end{array}$ \\
\hline Cordillera Azul & 1.25 & 1.74 & 1.47 & 2.05 \\
\hline Sierra del Divisor & 0.76 & 1.22 & 0.90 & 1.44 \\
\hline Alto Mayo & 3.61 & 3.91 & 4.25 & 4.60 \\
\hline Amarakaeri & 1.43 & 1.79 & 1.69 & 2.10 \\
\hline Tambopata & 1.85 & 1.96 & 2.17 & 2.30 \\
\hline $\begin{array}{l}\text { Ichigkat Muja- Cordillera } \\
\text { del condor }\end{array}$ & 0.40 & 0.40 & 0.55 & 0.55 \\
\hline Cordillera de Colan & 0.49 & 0.69 & 0.67 & 0.94 \\
\hline Santiago Comaina & 0.92 & 0.86 & 1.09 & 1.01 \\
\hline El Sira & 0.34 & 0.38 & 0.40 & 0.44 \\
\hline Bahuaja Sonene & 0.21 & 0.23 & 0.25 & 0.27 \\
\hline San Matías San Carlos & 0.67 & 1.10 & 0.78 & 1.29 \\
\hline Manu & 0.02 & 0.06 & 0.03 & 0.08 \\
\hline Chayu Naín & 0.70 & 0.73 & 0.95 & 0.99 \\
\hline Pampa Hermosa & 0.10 & 0.16 & 0.14 & 0.22 \\
\hline Yanachaga-Chemillén & 0.10 & 0.18 & 0.12 & 0.21 \\
\hline Gueppy & 0.00 & 0.01 & 0.01 & 0.02 \\
\hline Pagaibamba & 0.11 & 0.10 & 0.20 & 0.18 \\
\hline Rio Abiseo & 0.00 & 0.01 & 0.00 & 0.01 \\
\hline Pui Pui & 0.00 & 0.01 & 0.00 & 0.01 \\
\hline Megantoni & 0.00 & 0.01 & 0.00 & 0.01 \\
\hline Pacaya Samiria & 0.00 & 0.00 & 0.00 & 0.00 \\
\hline Tingo María & 0.00 & 0.01 & 0.00 & 0.01 \\
\hline Alto Purus & 0.00 & 0.00 & 0.00 & 0.00 \\
\hline Huascarán & 0.00 & 0.00 & 0.00 & 0.00 \\
\hline Machiguenga & 0.00 & 0.00 & 0.00 & 0.00 \\
\hline
\end{tabular}

Fuente: Elaboración propia en base a los datos estimados. 
Los valores de la relación B/C para Ef (75\%) y $\mathrm{Pr}=$ US $\$ 10.00$ oscilan entre 4.25 y 0 cuando la tasa de descuento fue del $10 \%$. Bajo ese escenario, sería viable implementar actividades REDD+ en 5 ANP: BP Alto Mayo, RN Tambopata, RC Amarakaeri, el PN Cordillera Azul y ZR Santiago Comaina. Por otro lado, cuando la tasa de descuento utilizada es de $5 \%$ los valores de la relación $\mathrm{B} / \mathrm{C}$ oscilan entre 4.6 y 0 . Bajo ese escenario sería viable implementar actividades REDD+ en 7 ANP: las 5 anteriormente mencionadas más ZR Sierra Divisor, y BP San Matías-San Carlos.

Los resultados obtenidos por el análisis son corroborados por el interés actual por parte de diferentes actores públicos y privados en implementar actividades REDD+ en algunas de las ANP del área de estudio. La evidencia muestra que las iniciativas de proyectos REDD+ en las ANP se desarrollan en las que presentan relación costo beneficio más altas, como son: Selva central que incluye tres parques Yanachaga-Chemillen, Yanesha y San Matías-San Carlos (Scriven 2012), Tambopata (Hajek, Ventresca et al. 2011), la iniciativa Mat nu-Amarakaeri (Hajek, Ventresca et al. 2011),
Cordillera Azul (http://www.cima.org.pe/not_ archivos_det.php?n=38) y Alto Mayo (http:// www.conservation.org/global/peru/iniciativas_actuales/Pages/ICAM.aspx).

Como se mencionó en la metodología, se consideró conveniente construir un escenario que incluye tanto los costos de oportunidad (CO) como los costos de transacción (CT). Para incluir los costos mencionados, se seleccionaron las ANP que presenten relación B/C mayor a 1 bajo el escenario que considera $\mathrm{Pr}=$ US\$ 10.0, Ef (75\%) y tasa de descuento del $5 \%$. Los costos de oportunidad fueron estimados con base al valor promedio por ha reportado por Armas et al. (2009) equivalente a 1515.82 US\$/ ha.

Los resultados fueron estimados, incluyendo los costos de oportunidad y transacción, para $\mathrm{Pr}=$ US\$ 5.4, Ef (75\% y 100\%) y tasa de descuento del $5 \%$. Se muestran que de incluirse los costos de oportunidad y de transacción bajo las condiciones descritas anteriormente no habría ningún ANP que haría viable implementar REDD+ (ver Tabla 5).

Tabla 5. Valores de la relación $\mathrm{B} / \mathrm{C}$ para $\mathrm{Pr}=$ US\$ 5.4 incluyendo costos de oportunidad y costos de transacción

\begin{tabular}{|l|c|c|c|c|}
\hline \multicolumn{1}{c}{ ANP } & \multicolumn{2}{c}{ B/C (100\%) } & \multicolumn{2}{c}{ B/C (75\%) } \\
\cline { 2 - 5 } & Tasa Descuento & Tasa Descuento & Tasa Descuento & Tasa Descuento \\
\hline Cordillera Azul & $\mathbf{1 0 \%}$ & $\mathbf{5 \%}$ & $\mathbf{1 0 \%}$ & $\mathbf{5 \%}$ \\
Sierra del Divisor & 0.36 & 0.43 & 0.40 & 0.47 \\
Alto Mayo & 0.25 & 0.34 & 0.28 & 0.37 \\
Amarakaeri & 0.26 & 0.32 & 0.28 & 0.34 \\
Tambopata & 0.26 & 0.32 & 0.29 & 0.35 \\
Santiago Comaina & 0.24 & 0.27 & 0.25 & 0.29 \\
San Matías San Carlos & 0.16 & 0.17 & 0.17 & 0.19
\end{tabular}

Fuente: Elaboración propia en base a los datos estimados. 
Asimismo, si los costos de oportunidad fueran disminuidos en US\$ 1 000, los resultados muestran que se mantendría la situación anterior, en la que en ninguna ANP sería viable implementar REDD+. La situación varía lige- ramente cuando se incrementa el Pr de 5.4 a 11. 7 USS (ver Tabla 6). Dado ese escenario, sería viable implementar REDD+ en una ANP, Cordillera Azul.

Tabla 6. Valores de la relación B/C para Pr = US\$ 11.7 incluyendo costos de oportunidad y transacción

\begin{tabular}{|c|c|c|c|c|}
\hline \multirow[b]{2}{*}{ ANP } & \multicolumn{2}{|c|}{$B / C(100 \%)$} & \multicolumn{2}{|c|}{$\mathrm{B} / \mathrm{C}(75 \%)$} \\
\hline & $\begin{array}{c}\text { Tasa Descuento } \\
10 \%\end{array}$ & $\begin{array}{c}\text { Tasa Descuento } \\
5 \%\end{array}$ & $\begin{array}{c}\text { Tasa Descuento } \\
10 \%\end{array}$ & $\begin{array}{c}\text { Tasa Descuen- } \\
\text { to } 5 \%\end{array}$ \\
\hline Cordillera Azul & 0.79 & 0.94 & 0.86 & 1.01 \\
\hline Sierra del Divisor & 0.55 & 0.74 & 0.60 & 0.80 \\
\hline Alto Mayo & 0.57 & 0.70 & 0.60 & 0.74 \\
\hline Amarakaeri & 0.57 & 0.70 & 0.62 & 0.75 \\
\hline Tambopata & 0.51 & 0.59 & 0.55 & 0.64 \\
\hline Santiago Comaina & 0.34 & 0.37 & 0.37 & 0.41 \\
\hline San Matías San Carlos & 0.22 & 0.35 & 0.25 & 0.40 \\
\hline
\end{tabular}

Fuente: Elaboración propia en base a los datos estimados.

\section{CONCLUSIONES}

Según el análisis realizado, bajo supuestos moderados, el número de ANP en las que sería viable la implementación de proyectos REDD+, según las condiciones actuales del mercado voluntario de carbono forestal, varía apenas entre 2 y 7 . Dependerá de: (i) el precio de los VERs; (ii) del aprovechamiento de economías de escala; y (iii) de la tasa de descuento que se utilice. Según el análisis, las ANP que debieran priorizarse para la implementación de REDD+ por presentar los valores de la relación $B / C$ más altos son: $B P$ Alto Mayo, RN Tambopata, RC Amarakaeri, PN Cordillera Azul, ZR Santiago Comaina, ZR Sierra Divisor, BP San Matías-San Carlos.

En las ANP del SINAMPE que no presentan altas amenazas de deforestación y/o no tie- nen alto contenido de carbono almacenado, no será viable la implementación de REDD+ a escala de proyecto. El análisis confirma que los ingresos potenciales por ventas de VERs forestal varían según: las amenazas de deforestación, las reservas de carbono, el nivel de eficiencia de las actividades REDD+ a implementarse y los precios de los VERs. Con ello, se demuestra la necesidad de diseñar una estrategia REDD+ a ser implementada en el SINAMPE que tenga un alcance nacional y que considere otros mecanismos financieros alternativos para dotar de recursos financieros óptimos a estas ANP.

Los resultados confirman que los costos de implementar actividades REDD+ efectivas varían según la ANP. Estas no necesariamente están relacionadas con variaciones en los 
contenidos de carbono, más bien se refieren a aquellas con el tamaño de las ANP y con las actividades a implementarse. Por ello, para REDD+ a escala de proyecto será necesario identificar las áreas que presenten mayor costo efectividad en términos de EGEI (a escala nacional) o mayor costo beneficio (a escala de proyecto).

Al considerar los costos de oportunidad y los costos de transacción En el presente análisis, los resultados muestran que REDD+ no es viable en ningún ANP bajo los supuestos definidos. Sin embargo, si el precio de los VERs es incrementado hasta un valor equivalente a US\$ 11.7, sería viable implementar REDD+ solo en la PN Cordillera Azul si es que se usara una tasa de descuento del $5 \%$ y un escenario Ef (75\%).

El estudio sugiere que REDD+, por sí mismo, no es una alternativa viable para dotar de sostenibilidad financiera a las ANP del Perú. Sin embargo, este mecanismo puede complementar mecanismos de conservación existentes no solo en ANP si no también en tierras indígenas. Asimismo, los pagos de carbono, puede ser un incentivo que incremente la viabilidad de realizar actividades de manejo forestal sostenible en concesiones forestales. 


\section{REFERENCIAS BIBLIOGRÁFICAS}

Angelsen, A., Ed. (2008). Moving Ahead with REDD: Issues, Options and Implications. Bogor, Indonesia, CIFOR.

Angelsen, A., Brown, S., Cyril, L., Peskett, L. and Streck, C. (2009). Reducing Emissions from Deforestation and Forest Degradation (REDD): An Option Assessment Report, Meridian Institute.

Baker, T. R., Jones, J. P. G., Rendón Thompson, O. R., Cuesta, R. M. R., Del Castillo, D., Aguilar, I. C., Torres, J. and Healey, J. R. (2010). "How can ecologists help realise the potential of payments for carbon in tropical forest countries?" Journal of Applied Ecology 47(6): 1159-1165.

Bellassen, V. and Gitz, V. (2008). "Reducing Emissions from Deforestation and Degradation in Cameroon - Assessing costs and benefits." Ecological Economics 68(1-2): 336344.

Börner, J. and Wunder, W. (2008). "Paying for avoided deforestation in the Brazilian Amazon: from cost assessment to scheme design." International Forestry Review 10(3): 496-511.

Börner, J., Wunder, S., Wertz-Kanounnikoff, S., Tito, M.R., Pereira, L. and Nascimento, N. (2010). "Direct conservation payments in the Brazilian Amazon: Scope and equity implications." Ecological Economics 69(6): 1272-1282.

Burgess, N. D., Bahane, B., Clairs, T., Danielsen,
F., Dalsgaard, S., Funder, M., Hagelberg, N., Harrison, P., Haule, C., Kabalimu, K., Kilahama, F., Kilawe, E., Lewis, S. L., Lovett, J.C., Lyatuu, G. Marshall, A.R., Meshack, C., Miles, L., Milledge, S. A. H., Munishi, P. K. T., Nashanda, E., Shirima, D., Swetnam, R.D., Willcock, S., Williams, A. and Zahabu, E. (2010). "Getting ready for REDD+ in Tanzania: a case study of progress and challenges." Oryx 44(03): 339-351.

Da Fonseca, G. A. B., Rodriguez, C. M., Midgley, G., Busch, J., Hannah, L. and Mittermeier, R. A. (2007). “No Forest Left Behind.” PLoS Biol 5(8): e216.

Diaz, D., Hamilton, K. and Johnson, E. (2011). El Estado de los Mercados de Carbono Forestal 2011: Desde el Dosel al Dólar. http://www. forest-trends.org/documents/files/doc_3052. pdf, Forest Trends: 79.

Ebeling, J. and Yasué, M. (2008). "Generating carbon finance through avoided deforestation and its potential to create climatic, conservation and human development benefits." Philosophical Transactions of the Royal Society B: Biological Sciences 363(1498): 19171924.

Hajek, F., Ventresca, M. J., Scriven, J. and Castro, A. (2011). "Regime-building for REDD+: Evidence from a cluster of local initiatives in south-eastern Peru." Environmental Science \&amp; Policy 14(2): 201-215.

Hiraldo, R. and Tanner, T. (2011). "Forest Voices: Competing Narratives over REDD+." IDS Bulletin 42(3): 42-51. 
Hunt, C. (2010). "The costs of reducing deforestation in Indonesia." Bulletin of Indonesian Economic Studies 46(2): 187-192.

Irawan, S., Tacconi, L. and Ring, I. (2013). "Stakeholders' incentives for land-use change and REDD+: The case of Indonesia." Ecological Economics 87(0): 75-83.

Kremen, C., Niles, J. O., Dalton, M. G., Daily, G. C., Ehrlich, P. R., Fay, J. P., Grewal, D. and Guillery, R. P. (2000). "Economic Incentives for Rain Forest Conservation Across Scales." Science 288(5472): 1828-1832.

Malhi, Y., Roberts, J. T., Betts, R. A., Killeen, T. J., Li, W. and Nobre, C. A. (2008). "Climate Change, Deforestation, and the Fate of the Amazon." Science 319(5860): 169-172.

Merger, E., Held, C., Tennigkeit, T. and Blomley, T. (2012). "A bottom-up approach to estimating cost elements of REDD+ pilot projects in Tanzania." Carbon Balance and Management 7.

Miles, L. and Kapos, V. (2008). "Reducing Greenhouse Gas Emissions from Deforestation and Forest Degradation: Global Land-Use Implications." Science 320(5882): 1454-1455.

MINAM (2010). Segunda Comunicación de Nacional del Perú a la Convención Marco de las Naciones Unidas Para el Cambio Climático 2010. MINAM. Lima - Peru, Impresiones y ediciones Aguilar S.A.C: 200.

MINAM (2011). Readiness Preparation Proposal ${ }^{\circ}$
Nepstad, D., Soares-Filho, B. S., Merry, F., Lima, A., Moutinho, P., Carter, J., Bowman, M., Cattaneo, A., Rodrigues, H., Schwartzman, S., McGrath, D. G., Stickler, C. M., Lubowski, R., Piris-Cabezas, P., Rivero, S., Alencar, A., Almeida, O. and Stella, O. (2009). "The End of Deforestation in the Brazilian Amazon." Science 326(5958): 1350-1351.

Pagiola, S. and Bosquet, B. (2009). Estimating the Costs of REDD at the Country Level, World Bank.

Scriven, J. N. H. (2012). "Preparing for REDD: Forest Governance Challenges in Peru's Central Selva." Journal of Sustainable Forestry 31(4-5): 421-444.

Stern, N. (2007). El informe Stern: La verdad del cambio climático. Barcelona-España, Paidós Ibérica.

Streck, C. (2012). "Financing REDD+: matching needs and ends." Current Opinion in Environmental Sustainability 4(6): 628-637.

Tacconi, L. (2012). "Redefining payments for environmental services." Ecological Economics 73(0): 29-36.

Turner, W. R., Brandon, K., Brooks, T. M., Costanza, R., Da Fonseca, G. and Portela, R. (2007). "Global Conservation of Biodiversity and Ecosystem Services." BioScience 57(10): 868873.

Turner, W. R., Brandon, K., Brooks, T. M., Gascon, C., Gibbs, H. K., Lawrence, K. S., Mitter- 
meier, R. A. and Selig, E. R. (2012). "Global Biodiversity Conservation and the Alleviation of Poverty." BioScience 62: 85-92.

UNFCCC (2009). Report of the Conference of the Parties on its fifteenth session. Conference of the Parties on its fifteenth session. Copenhague, Dinamarca.

UNFCCC (2010). Decisiones adoptadas por la Conferencia de las Partes. Conferencia de las Partes. Cancun, Mexico.

UNFCCC (2011). Report of the Conference of the Parties on its seventeenth session - FCCC/ CP/2011/9/Add.1. Conference of the Parties. Durban, South Africa.

Van der Werf, G. R., Morton, D. C., DeFries, R. S., Olivier, J. G. J., Kasibhatla, P. S., Jackson, R. B., Collatz, G. J. and Randerson, J. T. (2009). “CO2 emissions from forest loss." Nature Geosci 2(11): 737-738.

Villanueva, J. P., Lima-Peru. , Ed. ( 2005). Análisis de las necesidades de financiamiento 2005-2014. Lima, Peru, PROFONANPE.

Wertz-Kanounnikoff, S. (2008). Estimating the costs of reducing forest emissions: A review of methods, Center for International Forestry Research (CIFOR). 


\section{ANEXOS}

\section{Anexo 1. Proceso para estimar las emisiones de GEI para el ANP Alto Mayo}

\begin{tabular}{|c|c|c|c|c|c|c|c|c|c|}
\hline \multirow{2}{*}{$\begin{array}{l}\text { Contenido } \\
\mathrm{CO}_{2}\left(\mathrm{tCO}_{2}\right)\end{array}$} & \multicolumn{9}{|c|}{ Superficie deforestada por contenido de $\mathrm{CO}_{2}$} \\
\hline & 2010 & 2015 & 2020 & 2025 & 2030 & 2035 & 2040 & 2045 & 2050 \\
\hline 25 & - & - & - & - & - & - & - & - & - \\
\hline 50 & 6 & 8 & - & - & - & - & 20 & & 45 \\
\hline 75 & 582 & 903 & 202 & 59 & - & - & 67 & 120 & 402 \\
\hline 100 & 691 & 839 & 384 & 28 & 41 & 145 & 355 & 322 & 328 \\
\hline 150 & 1,339 & 1,306 & 1,511 & 351 & 453 & 790 & 1,027 & 787 & 2,462 \\
\hline 200 & 76 & 16 & 22 & 10 & 269 & 87 & 470 & 460 & 1,036 \\
\hline 250 & 5,755 & 4,862 & 3,408 & 1,739 & 2,779 & 2,010 & 3,966 & 4,476 & 5,180 \\
\hline 300 & 1,486 & 1,445 & 1,151 & 356 & 637 & 468 & 103 & 677 & 1,500 \\
\hline 350 & - & 0 & 0 & 0 & - & - & - & - & - \\
\hline 400 & & - & - & - & - & - & - & - & - \\
\hline 400 a más & & - & - & - & - & - & - & - & - \\
\hline Total $\mathrm{CO}_{2}\left(\mathrm{tCO}_{2}\right)$ & $2,213,510$ & 200,290 & $1,481,811$ & 603,340 & $1,011,837$ & 793,062 & $1,311,874$ & $1,573,110$ & $2,386,689$ \\
\hline
\end{tabular}

Fuente: Elaboración propia con base en Soares-Filho et al. (2006) y Saatchi et al. (2007). 


\section{Anexo 2. EGEI totales proyectadas al 2050 por ANP bajo el escenario BAU}

\begin{tabular}{|c|c|}
\hline \multirow{2}{*}{ ANP } & EGEI en $\mathrm{tCO}_{2}(\mathrm{BAU})$ \\
\hline & Total \\
\hline Cordillera Azul & $45,700,135.50$ \\
\hline Sierra del Divisor & $41,674,205.50$ \\
\hline Alto Mayo & $13,375,522.00$ \\
\hline Amarakaeri & $12,783,379.25$ \\
\hline Tambopata & $9,061,489.50$ \\
\hline Ichigkat Muja-Cordillera del Cóndor & $6,815,232.75$ \\
\hline Cordillera de Colan & $6,078,842.50$ \\
\hline Santiago Comaina & $5,682,639.00$ \\
\hline El Sira & $4,246,340.00$ \\
\hline BahuajaSonene & $4,109,941.00$ \\
\hline San Matias-San Carlos & $3,823,348.50$ \\
\hline Manu & $3,688,125.25$ \\
\hline ChayuNaín & $2,916,775.25$ \\
\hline Pampa Hermosa & $536,393.00$ \\
\hline Yanachaga-Chemillén & $482,424.00$ \\
\hline Gueppy & $262,992.25$ \\
\hline Pagaibamba & $204,497.50$ \\
\hline Río Abiseo & $132,686.00$ \\
\hline PuiPui & $126,472.75$ \\
\hline Megantoni & $120,147.75$ \\
\hline PacayaSamiria & $91,809.50$ \\
\hline Tingo Maria & $52,379.00$ \\
\hline Alto Purus & $3,968.65$ \\
\hline Huascarán & $1,140.75$ \\
\hline Machiguenga & -4.75 \\
\hline
\end{tabular}

Fuente: Elaboración propia con base en Soares-Filho et al. (2006) y Saatchi et al. (2007). 